\title{
Uso Prático de um Índice de Risco de Complicações após Cirurgia Cardíaca
}

\author{
Practical Use of a Risk Assessment Model for Complications after Cardiac Surgery \\ Tânia Mara Varejão Strabelli, Noedir Antonio Groppo Stolf, David Everson Uip \\ Instituto do Coração do Hospital das Clínicas - Faculdade de Medicina da Universidade de São Paulo - São Paulo, SP - Brasil
}

\section{Resumo}

Fundamento: Identificar os fatores de risco para complicações pós-operatórias do paciente cardiopata com indicação cirúrgica que podem influenciar na decisão sobre a conduta terapêutica.

Objetivo: Descrever a experiência de um hospital de Cardiologia na validação e uso prático de um escore de risco pré-operatório.

Métodos: Para validação do escore escolhido (Tuman), avaliaram-se consecutiva e prospectivamente 300 pacientes adultos antes da cirurgia cardíaca eletiva com o uso de circulação extracorpórea (CEC). Pacientes com escore de 0 a 5 foram considerados de baixo risco; de 6 a 9 como risco moderado; e maior que 10, como alto risco para complicações cardíacas, infecciosas, neurológicas, pulmonares e renais, além de óbito.

Resultados: A classificação de Tuman mostrou relação estatisticamente significante com ocorrência de complicações infecciosas $(p=0,010)$, com outras complicações pós-operatórias $(p=0,034)$ e com evolução para óbito ( $p<0,001)$. Infecção pulmonar foi a mais freqüente dentre as complicações infecciosas $(\mathbf{1 5 , 3 \% )}$, Os pacientes infectados tiveram maior tempo de permanência na UTI $(p=0,001)$ e internação mais prolongada $(p=0,001)$. Após o uso rotineiro, uma nova avaliação de 154 pacientes operados em 2005 confirmou a validade desse escore na identificação daqueles com maior risco de infecções pós-operatórias.

Conclusão: Escolheu-se o escore de Tuman por envolver variáveis de fácil obtenção, por classificar no mesmo sistema as cirurgias mais freqüentemente realizadas e prever risco de complicações pós-operatórias, além da mortalidade. Seu uso continuado nesse hospital permitiu identificar o grupo de pacientes com maior risco de complicações, especialmente as infecciosas, mas não foi preciso na predição do risco individual. (Arq Bras Cardiol 2008; 91(5) : 342-347)

Palavras-chave: Cirurgia torácica, complicações pós-operatórias, risco, infecção.

\section{Summary}

Background: The identification of risk factors for postoperative complications in cardiac patients with surgical indication may influence the therapeutic decision.

Objective: To describe the experience of a Cardiology hospital in the validation and practical use of a preoperative risk score.

Methods:To validate TUMAN's score, chosen by considering morbidity and mortality, 300 adult patients were prospectively evaluated before elective cardiac surgery with the use of extracorporeal circulation (ECC). Patients with a score of zero to five were considered as being low risk; from six to nine, as moderate risk and a score higher than 10 as high risk for cardiac, infectious, neurological, pulmonary and renal complications, as well as death.

Results: The TUMAN classification showed a statistically significant association with the occurrence of infectious complications $(p=0.010)$, with the other postoperative complications $(p=0.034)$ and death $(p<0.001)$. Pulmonary infection was the most frequent infectious complication $(15.3 \%)$ and Infected patients had a longer ICU stay duration $(p=0.001)$ and more prolonged hospitalization $(p=0001)$. After routine use, a new review of 154 patients operated in 2005, confirmed the validity of this score in the identification of those with the highest risk of postoperative infections.

Conclusion: TUMAN's score was chosen as it uses variables that can be promptly obtained, classifies in the same system the most frequently performed surgeries and predicts risk of postoperative complications, in addition to mortality. Its continued use in this hospital has been able to identify the group of patients with increased risk of complications, especially infectious ones, although it was not useful in the prediction of individual risk. (Arq Bras Cardiol 2008; 91(5) : 315-320)

Key words: Thoracic surgery; postoperative complications; risk; infection.

Full texts in English - http://www.arquivosonline.com.br

Correspondência: Tânia Mara Varejão Strabelli •

Rua Voluntários da Pátria, 2.831, ap.151 Cep: 02401-100, São Paulo,SP - Brasil

E-mail: tania.strabelli@incor.usp.br

Artigo enviado em 26/12/2007; revisado recebido em 07/01/2008; aceito em 31/01/2008 


\section{Introdução}

Nas últimas duas décadas, houve uma mudança significativa no perfil dos pacientes submetidos a cirurgia cardíaca em razão do aperfeiçoamento dos métodos diagnósticos e terapêuticos. A cirurgia de revascularização do miocárdio, por exemplo, está sendo indicada mais tardiamente ou em pacientes com lesões mais graves, resultando em maior número de situações de risco, como reoperações, doenças associadas (diabetes, hipertensão arterial, nefropatia) e pacientes mais idosos.

Em razão dos custos crescentes dos serviços de saúde e do maior número de opções para o tratamento das cardiopatias, a identificação dos fatores de risco do paciente para complicações pós-operatórias pode influenciar a decisão sobre a conduta mais adequada. Além disso, para as organizações financiadoras dos procedimentos, é importante definir parâmetros de risco de complicações para aprimorar o planejamento dos recursos necessários e o custo final da internação.

Desse modo, é importante identificar, no período préoperatório, os pacientes com maior risco de complicações pós-operatórias. Dentre essas, a infecção continua sendo uma das maiores causas de morbidade e mortalidade em pacientes cirúrgicos, havendo um interesse especial em identificar fatores de risco para sua ocorrência.

Vários estudos têm sido publicados para comparar os diversos índices de risco em uso por instituições em todo o mundo ${ }^{1-4}$, e a maioria deles utilizou variáveis semelhantes. Além disso, esses dados têm sido utilizados para predizer o tempo de internação na unidade de terapia intensiva (UTI), permitindo racionalizar o programa cirúrgico ${ }^{5}$ e implementar as medidas profiláticas pertinentes. Entretanto, há pouca referência às complicações infecciosas.

Descrevemos a experiência de um hospital universitário de Cardiologia na validação e no uso prático de um escore de risco para complicações, especialmente infecções, e óbito.

\section{Métodos}

Para validação do escore de risco escolhido $\left(\operatorname{Tuman}^{6}\right)$, foram avaliados consecutiva e prospectivamente 300 pacientes adultos internados para serem submetidos a cirurgia cardíaca eletiva com o uso de circulação extracorpórea (CEC), do tipo revascularização do miocárdio (RM) aneurismectomia de venrtrículo esquerdo associada e/ou procedimento valvar (implante de prótese ou cirurgia conservadora). O trabalho foi aprovado pela Comissão de Ética e Pesquisa do Hospital das Clínicas da Faculdade de Medicina da Universidade de São Paulo.

Na tabela 1 estão descritas as variáveis utilizadas nesse escore, obedecendo às seguintes definições:

- Infarto agudo do miocárdio: paciente apresentando dois ou mais dos seguintes achados: elevação da creatinofosfoquinase-fração de membrana (CKMB); ondas Q novas ao eletrocardiograma; falhas na captação do pirofosfato de tecnécio na cintilografia do miocárdio.

- Hipertensão pulmonar: pressão da artéria pulmonar 25 milímetros de mercúrio $(\mathrm{mmHg})$ acima da pressão arterial sistêmica, evidenciada ao ecocardiograma ou durante estudo hemodinâmico.

- Doença cerebrovascular: antecedente de acidente vascular cerebral e/ou alterações vasculares diagnosticadas durante carotidoangiografia.

- Insuficiência cardíaca congestiva: alterações radiológicas compatíveis com congestão pulmonar ou presença de terceira bulha cardíaca (B3).

- Disfunção de ventrículo esquerdo: fração de ejeção menor que 35\% ao ecocardiograma.

- Disfunção renal: creatinina sérica maior que 1,4 mg/dl.

Quanto à classificação de risco de complicações, pacientes com escore de 0 a 5 foram considerados de baixo risco; escore de 6 a 9, risco moderado; e escore maior que 10, alto risco.

Quanto ao diagnóstico da cardiopatia de base, os pacientes foram subdivididos em três grupos: insuficiência coronariana (ICO); cardiopatia valvar (VAL) ou associação dos dois diagnósticos (ICO + VAL).

A cefuroxima (via venosa) foi utilizada como antibioticoprofilaxia (1,5 grama iniciado uma hora antes da cirurgia e repetido a cada doze horas, totalizando cinco doses).

Foram computadas as complicações pós-operatórias que ocorreram durante a internação hospitalar, de acordo com os critérios a seguir relacionados:

Complicações cardíacas: infarto agudo do miocárdio perioperatório: paciente apresentando dois ou mais dos seguintes: elevação da CKMB; ondas Q novas ao eletrocardiograma; falhas na captação do pirofosfato de tecnécio na cintilografia do miocárdio; síndrome de baixo débito cardíaco: índice cardíaco menor que dois litros por minuto por metro quadrado com necessidade de drogas inotrópicas por mais de duas horas e/ou uso de balão intra-aórtico.

\section{Tabela 1 - Sistema de escore clínico de risco, segundo Tuman e cols. ${ }^{6}$}

\begin{tabular}{ll}
\hline Fator pré-operatório & Escore \\
\hline Cirurgia de emergência & 4 \\
\hline Idade & 1 \\
\hline 65-74 anos & 2 \\
\hline$>75$ anos & 2 \\
\hline Disfunção renal & \\
\hline Infarto do miocárdio prévio & 1 \\
\hline 3-6 meses & 2 \\
\hline$<3$ meses & 2 \\
\hline Sexo feminino & 2 \\
\hline Cirurgia cardíaca prévia & 2 \\
\hline Hipertensão pulmonar & 2 \\
\hline Doença cerebrovascular & 2 \\
\hline Troca valvar múltipla ou RM + troca valvar & 1 \\
\hline Troca de valva aórtica ou mitral & 1 \\
\hline Insuficiência cardíaca congestiva & 1 \\
\hline Disfunção de ventrículo esquerdo & 2 \\
\hline
\end{tabular}


Complicações pulmonares: intubação traqueal ou ventilação mecânica por mais de 48 horas após a cirurgia; necessidade de reintubação traqueal associada à ventilação mecânica.

Complicações renais: nível sérico de creatinina dois miligramas por decilitro (mg/dl) acima do nível pré-operatório; necessidade de diálise em qualquer momento do pósoperatório.

Complicações neurológicas: alteração do nível de consciência ou coma ocorrendo em associação com lesão neurológica durante a cirurgia; alteração sensorial, motora ou de reflexos em qualquer momento do pós-operatório.

Complicações infecciosas: as infecções foram classificadas de acordo com as definições do Center for Diseases Control ${ }^{7}$, adaptadas pela Sub-Comissão de Controle de Infecção Hospitalar do InCor. Foram computadas as infecções pulmonares, urinárias, da ferida operatória (tórax e membros inferiores) e as infecções da corrente sangüínea.

As variáveis-resposta avaliadas foram complicações pós-operatórias, complicações infecciosas e óbito. A comparação de proporções dessas variáveis entre grupos de pacientes estabelecidos a partir da exposição ou não a fatores pré-operatórios estudados foi avaliada pelo teste de Qui-quadrado ou teste exato de Fisher. O risco relativo de cada resposta com o correspondente intervalo de confiança de $95 \%$ foi estimado para cada variável. O nível de significância estabelecido para análise foi 5\%. O sistema SAS - Statistical Analysis System - foi empregado para a realização dos cálculos estatísticos.

\section{Resultados}

No estudo de validação, quatro pacientes foram excluídos: um faleceu antes da cirurgia, dois apresentaram contraindicações para a operação, recebendo tratamento clínico, e um paciente foi submetido somente à aneurismectomia de ventrículo esquerdo. Os dados pré-operatórios referem-se, portanto, a 296 pacientes, com idades variando de 20 a 84

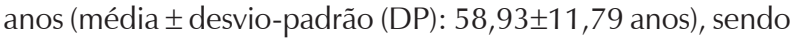
$208(70,3 \%)$ do sexo masculino.

Duzentos e dez pacientes estavam no grupo ICO, 59 no grupo VAL e 27 no ICO + VAL. Ocorreram 57 procedimentos de troca valvar (31 aórtica; 26 mitral; sete associações), 17 plastias e nove comissurotomias; 232 revascularizações do miocárdio e 23 aneurismectomias de ventrículo esquerdo.

O escore de complicações de Tuman $^{6}$ mostrou relação estatisticamente significante com ocorrência de complicações infecciosas $(p=0,010)$, com a de outras complicações pósoperatórias $(p=0,034)$ e com evolução para óbito $(p<0,001)$ como demonstrado na tabela 2. A incidência de complicações estimada pelos autores seria de $14,3 \%$ a $14,8 \%$ para baixo risco, $33,8 \%$ a $35 \%$ para risco moderado, e $59,4 \%$ a $62,5 \%$ para alto risco. Apesar de as taxas encontradas serem superiores às esperadas, houve relação estatisticamente significante entre o escalonamento dos riscos e a maior ocorrência de complicações ( $p=0,034)$.

A incidência de cada tipo de complicação pós-operatória de acordo com o mesmo escore está descrita na tabela 3. Dos 296 pacientes, dois foram excluídos dessa análise pois faleceram durante a operação, resultando em 294 pacientes estudados

Cento e doze pacientes $(38,1 \%)$ apresentaram pelo menos uma complicação pós-operatória, com predomínio das cardíacas (72 pacientes - 24,5\%) e infecciosas (69 pacientes - 23,5\%), mesmo após estratificação por cardiopatia de base. Seguiram-se as pulmonares (33 pacientes - 11,2\%), neurológicas (32 pacientes $-10,5 \%$ ) e renais (9 pacientes $-3,1 \%)$.

O risco relativo estimado de complicações no grupo ICO foi 1,5 vez maior que no grupo VAL, e no grupo ICO $+V A L$, 1,8 vez maior que no grupo VAL. Na análise detalhada da distribuição das complicações pós-operatórias, a complicação cardíaca isoladamente foi o evento mais freqüente (29 pacientes), seguido por infecção pós-operatória (22 pacientes) e complicação cardíaca e infecciosa associadas (10 pacientes). Complicação renal e pulmonar não ocorreram isoladamente. Seis pacientes apresentaram apenas complicação neurológica e nenhum deles evoluiu para óbito.

Infecção pulmonar foi a mais freqüente dentre as complicações infecciosas $(15,3 \%)$, seguida por infecção da corrente sanguínea $(3,1 \%)$, infecção da ferida operatória do

Tabela 2 - Incidência de complicações pós-operatórias, complicações infecciosas e óbito de acordo com escore de Tuman e cols. ${ }^{6}$

\begin{tabular}{|c|c|c|c|c|c|}
\hline Variável & Escore de Tuman & $\mathrm{n}$ & $\mathrm{p}$ & Risco relativo & $\begin{array}{c}\text { Intervalo de confiança } \\
95 \%\end{array}$ \\
\hline \multirow{3}{*}{$\begin{array}{l}\text { Complicações } \\
\text { pós-operatórias }\end{array}$} & $0-5$ & $89(35,3)$ & $0,034^{*}$ & 1,53 & $(1,19 ; 2,13)$ \\
\hline & $6-9$ & $21(53,8)$ & & 1,89 & $(0,83 ; 4,28)$ \\
\hline & $>=10$ & $2(66,7)$ & & & \\
\hline \multirow{3}{*}{ Complicações infecciosas } & $0-5$ & $52(20,6)$ & 0,010 & & \\
\hline & $6-9$ & $15(38,5)$ & & 1,86 & $(1,17 ; 2,97)$ \\
\hline & $>=10$ & $2(66,7)$ & & 3,23 & $(1,40 ; 7,45)$ \\
\hline \multirow{3}{*}{ Óbito } & $0-5$ & $9(3,5)$ & $<0,001$ & 0,05 & $(0,02 ; 0,15)$ \\
\hline & $6-9$ & $12(30,8)$ & & 8,68 & $(3,92 ; 19,25)$ \\
\hline & $>=10$ & $2(66,7)$ & & 18,82 & $(6,75 ; 52,47)$ \\
\hline
\end{tabular}


Tabela 3 - Relação entre escore de Tuman e cols. ${ }^{6}$ e a ocorrência de complicações pós-operatorias nos 294 pacientes avaliados

\begin{tabular}{|c|c|c|c|c|c|c|c|}
\hline \multirow{3}{*}{ Complicações } & \multicolumn{6}{|c|}{ Escore de Tuman } & \multirow[t]{3}{*}{$P$} \\
\hline & \multicolumn{2}{|c|}{$\begin{array}{c}\mathbf{0 - 5} \\
252 \text { pacientes }\end{array}$} & \multicolumn{2}{|c|}{$\begin{array}{c}6-9 \\
39 \text { pacientes }\end{array}$} & \multicolumn{2}{|c|}{$\begin{array}{c}\quad \geq 10 \\
\text { 3 pacientes }\end{array}$} & \\
\hline & $n$ & $\%$ & $\mathrm{n}$ & $\%$ & $\mathrm{n}$ & $\%$ & \\
\hline Cardíacas & 53 & $(21,0)$ & 17 & $(43,6)$ & 2 & $(66,7)$ & $0,002^{\star}$ \\
\hline Pulmonares & 20 & $(7,9)$ & 11 & $(28,2)$ & 2 & $(66,7)$ & $<0,001^{*}$ \\
\hline Renais & 3 & $(1,2)$ & 4 & $(10,3)$ & 2 & $(66,7)$ & $<0,001^{*}$ \\
\hline Neurológicas & 23 & $(9,1)$ & 7 & $(17,9)$ & 2 & $(66,7)$ & $0,002^{*}$ \\
\hline Infecciosas & 52 & $(20,6)$ & 15 & $(38,5)$ & 2 & $(66,7)$ & $0,010^{*}$ \\
\hline
\end{tabular}

* resultado estatisticamente significante.

tórax $(2,7 \%)$ e dos membros inferiores $(2,7 \%)$ e infecção do trato urinário (2,4\%). A distribuição das infecções de acordo com a cardiopatia de base está descrita na tabela 4. Os pacientes infectados tiveram maior tempo de permanência na UTI $(10,8 \pm 14,1$ dias $\times 3,0 \pm 1,6$ dias - $p=0,001)$ e tempo de internação mais prolongado $(40,7 \pm 23,7$ dias $\times 22,7 \pm 8,5$ dias $-p=0,001)$.

Vinte e três pacientes ( 16 do sexo masculino) evoluíram para óbito $(7,8 \%)$, e dois ocorreram durante a cirurgia. A taxa de letalidade por grupo foi 4,8\% (10 pacientes) no grupo ICO, 13,6\% (oito pacientes) no VAL e 18,5\% (cinco pacientes) no ICO + VAL. As idades variaram entre $30 \mathrm{e}$ 79 anos (62,2 $\pm 14,9$ anos). Os pacientes que faleceram apresentaram maior incidência de complicações pósoperatórias quando comparados aos que sobreviveram $(p<0,001)$.

Após a validação, esse escore foi implantado na rotina de avaliação pré-operatória dos pacientes adultos submetidos à cirurgia cardíaca no InCor.

Uma nova avaliação de 154 pacientes operados em 2005 confirmou a validade desse escore na identificação daqueles com maior risco de infecções pós-operatórias. Como houve apenas dois pacientes com escore $\geq 10$ (tabela 5), comparamos aqueles com risco baixo e moderado de complicações $(p=0,0013$; risco relativo $=2,35)$.

\section{DIscussão}

Os profissionais da área de Saúde que acompanham o pós-operatório de cirurgias cardíacas, seja qual for sua área de atuação, deparam algumas vezes com situações clínicas de extrema gravidade, longas evoluções pós-operatórias e, outras vezes, seqüelas importantes que comprometem a qualidade de vida do paciente.

A avaliação dos resultados pós-cirúrgicos é o primeiro passo para medir a qualidade do atendimento médico e, para que essa seja precisa, é fundamental diferenciar os pacientes por gravidade ${ }^{3}$. Os prestadores de serviço também têm interesse em conhecer esses resultados para optar pela cobertura dos procedimentos nos hospitais com menores índices de complicações, menor duração da internação e menor mortalidade $^{8}$. Conhecer o perfil de risco do paciente pode, contudo, ajudar na decisão terapêutica e permitir a adoção de estratégias individualizadas de prevenção de complicações ${ }^{9}$. Essas questões mostram a importância de se aferirem os dados com a maior precisão possível e continuadamente.

O uso de escores de classificação para definição do risco de complicações pós-operatórias em cirurgia cardíaca se iniciou no final da década de 1980 e até o momento não há consenso sobre o melhor método para avaliar risco de complicações e óbito, devendo ser escolhido de acordo com as necessidades específicas do serviço. Não se encontrou referência sobre o uso de escores de risco em serviços de cirurgia cardíaca no Brasil.

\section{Tabela 4 - Incidência de infecção pós-operatória nos 294 pacientes de acordo com a cardiopatia de base}

\begin{tabular}{|c|c|c|c|c|c|c|c|c|}
\hline \multirow{3}{*}{ Infecção } & \multicolumn{6}{|c|}{ Cardiopatia de base } & & \\
\hline & \multicolumn{2}{|c|}{ Ico } & \multicolumn{2}{|c|}{ Val } & \multicolumn{2}{|c|}{ Ico + Val } & \multicolumn{2}{|c|}{ Total } \\
\hline & $n=208$ & $\%$ & $\mathrm{~N}=59$ & $\%$ & $\mathrm{n}=27$ & $\%$ & $\mathrm{n}$ & $\%$ \\
\hline Pulmonar & 32 & $(15,4)$ & 9 & $(15,2)$ & 4 & $(14,8)$ & 45 & $(15,3)$ \\
\hline Urinária & 6 & $(2,9)$ & - & - & 1 & $(3,7)$ & 7 & $(2,4)$ \\
\hline \multicolumn{9}{|l|}{ Ferida operatória } \\
\hline tórax & 7 & $(3,4)$ & - & - & 1 & $(3,7)$ & 8 & $(2,7)$ \\
\hline membros inferiores & 6 & $(2,9)$ & - & - & 2 & $(7,4)$ & 8 & $(2,7)$ \\
\hline Corrente sanguínea & 4 & $(1,9)$ & 3 & $(5,1)$ & 2 & $(7,4)$ & 9 & $(3,1)$ \\
\hline
\end{tabular}


Tabela 5 - Incidência das infecções pós-operatórias em 154 pacientes operados em 2005 de acordo com o escore de Tuman e cols. ${ }^{6}$

\begin{tabular}{lccc}
\hline $\begin{array}{l}\text { Escore De } \\
\text { Tuman }\end{array}$ & $\begin{array}{c}\text { Número } \\
\text { Pacientes }\end{array}$ & $\begin{array}{c}\text { Complicações } \\
\text { Infecciosas(\%) }\end{array}$ & Óbito \\
\hline $0-5$ & 97 & $16(16,5 \%)$ & $5(5,2 \%)$ \\
\hline $6-9$ & 55 & $24(43,6 \%)$ & $9(16,4 \%)$ \\
\hline$>10$ & 2 & $1(50 \%)$ & $1(50 \%)$ \\
\hline
\end{tabular}

Escolheu-se o elaborado por Tuman e cols ${ }^{6}$ por envolver variáveis de fácil obtenção, classificar no mesmo sistema as cirurgias mais freqüentemente realizadas (revascularização do miocárdio e/ou troca valvar) e também prever risco de complicações pós-operatórias, além da mortalidade. Esses autores realizaram análise univariada dos fatores de risco préoperatórios em 3.156 operações e Identificaram 11 variáveis que receberam valores diferenciados. Obtiveram, desse modo, categorias de risco de complicações pós-operatórias. O aumento do escore de risco também esteve associado à maior freqüência individual de complicações e ao prolongamento do tempo de internação em UTI.

O uso continuado do escore de Tuman e cols. ${ }^{6}$ em nosso hospital permitiu identificar o grupo de pacientes com maior risco de complicações, mas não se mostrou preciso na predição do risco individual, já que tanto as taxas de complicações quanto as de óbito foram superiores às estimadas. As taxas de letalidade no InCor têm variado, nos últimos três anos, entre 1,9\% e 2,9\% na revascularização do miocárdio, 4,2\% e 5\% nos procedimentos valvares, e entre $5,6 \%$ e $13 \%$ nas cirurgias associadas (RM e procedimento valvar), segundo dados fornecidos pela Unidade de Controle do Paciente Cirúrgico.

Em se tratando das complicações infecciosas, a literatura é escassa. Alguns autores citam infecção apenas como uma das complicações pós-operatórias ${ }^{2,3,6,10}$, sem utilizar a classificação de risco pré-operatório como preditor de infecção como fizemos neste estudo. A infecção foi superada em freqüência apenas pelas complicações cardíacas no pós-operatório (tabela 3) e o escore de Tuman associou-se de modo estatisticamente significante com a ocorrência de complicações infecciosas. podendo ser utilizado para identificar os pacientes em que se deva reforçar as medidas preventivas, como ser operado pelo cirurgião mais experiente, diminuir o tempo de internação pré-operatória e/ou receber profilaxia antimicrobiana individualizada.

É importante ressaltar que os sistemas de classificação de risco não consideram a evolução intra-operatória, que pode ser um fator decisivo na evolução do paciente.

Analisando as publicações mais recentes sobre escore de risco em cirurgia cardíaca, conclui-se que não se tem um modelo ideal e de aplicação universal. O escore de Parsonnet e cols. ${ }^{11}$, um dos mais citados na literatura, foi útil para identificar os pacientes com permanência menor que 24 horas em unidade de terapia intensiva (UTI) no pós- operatório numa análise de 5.591 pacientes num hospital de Londres com poucos leitos de UTI ${ }^{5}$. Entretanto, quando aplicado em 8.210 pacientes operados em quatro hospitais na região Noroeste da Inglaterra para identificar risco de evolução para óbito, os dados foram superestimados ${ }^{12}$. Mais recentemente, Marius Berman e cols. ${ }^{13}$ utilizaram em Israel um escore simplificado de Bernstein-Parsonnet (BP) que inclui variáveis obtidas facilmente na beira do leito, comparado com o EuroSCORE ${ }^{14}$ que foi desenvolvido na Europa. Para essa população, que é bastante heterogênea quanto à origem, o escore BP apresentou maior poder de discriminação para predizer mortalidade.

Geissler e cols. ${ }^{8}$ aplicaram diferentes escores em 1.999 pacientes submetidos a cirurgia cardíaca com circulação extracorpórea num hospital alemão, porém conseguiram acompanhamento até 30 dias de pós-operatório em apenas 504 pacientes, avaliando mortalidade, uso de dispositivos mecênicos de suporte, hemodiálise ou hemofiltração, infarto do miocárdio, duração de ventilação mecânica e de internação na UTI. Dentre os escores de Parsonnet, Cleveland Clinic, French, EuroSCORE, Pons e Ontário Province Risk (OPR), o EuroSCORE foi o que apresentou os melhores valores prediditivos para morbidade e mortalidade. Os valores prediditivos para morbidade foram substancialmente menores que os de mortalidade para todos os escores.

A partir do banco de dados da Society of Thoracic Surgeons (STS) dos Estados Unidos, foi desenvolvido um modelo para predizer mortalidade e morbidade nos primeiros 30 dias de pós-operatório da revascularização do miocárdio, estudando 503.478 indivíduos. Baseando-se nessa análise, 87\% dos pacientes evoluíram sem nenhuma complicação no período pós-operatório.

Para avaliar as diferenças entre o EuroSCORE e o STS na capacidade de predizer a mortalidade após revascularização do miocárdio, foram avaliados, retrospectivamente, 3.125 pacientes consecutivos. Os autores concluíram que os dois escores foram bom preditores de mortalidade, com discreta vantagem para o STS ${ }^{15}$.

Em outro estudo ${ }^{1}$, aplicaram-se cinco escores diferentes (Parsonnet, Canadian, Cleveland, New York e Northern New England) a 1.135 pacientes submetidos a revascularização do miocárdio num hospital comunitário americano para avaliar acurácia na predição de óbito. Não houve diferença significativa entre eles, apesar de utilizarem variáveis distintas.

Um estudo comparativo entre quatro escores de risco de mortalidade após cirurgia cardíaca (Parsonnet modificado, TU, Tuman e APACHE II) em 331 pacientes mexicanos ${ }^{16}$. Apenas o escore de TU não teve boa discriminação, porém Parsonnet modificado e APACHE II tiveram melhor calibração. Os autores concluíram que esses dois modelos podem ser utilizados como preditores de mortalidade em cirurgia cardíaca no México.

Esses sistemas de estratificação de riscos podem ser utilizados para pesquisa, orientação individual de pacientes ou para racionalização do uso de recursos em hospitais. Devem ser revisados periodicamente, pois tanto as características dos pacientes quanto os recursos 
diagnósticos e terapêuticos podem sofrer mudanças no decorrer dos anos.

É importante ressaltar que, embora a probabilidade de complicação possa ser estimada para um paciente, não se tem certeza absoluta em relação à sua evolução. Muitas vezes, porém, os pacientes de maior risco são os que mais se beneficiam com a cirurgia ${ }^{17}$.

Esses exemplos demonstram a necessidade de se validar um escore antes de iniciar seu uso na prática diária. e que, para proceder à sua escolha, devem-se considerar as características da população que se pretende avaliar, como foi feito neste estudo.

\section{Referências}

1. Reed JF, Olenchock SA. Comparative analysis of risk-adjusted bypass surgery stratification models in a community hospital. Heart Lung. 2003; 32: 38390.

2. Pinna-Pintor $P$, Bobbio $M$, Colangelo $S$, Veglia F, Ciammaria M, Cuni D, et al. Inaccuracy of four coronary risk-adjusted models to predict mortality in individual patients. Eur J Cardiothorac Surg. 2002; 21: 199-204.

3. Shroyer AL, Coombs LP, Peterson ED, Eiken MC, DeLong ER, Chen A, et al. The Society of Thoracic Surgeons: 30-day operative mortality and morbidity risk models. Ann Thorac Surg. 2003; 75: 1856-64.

4. Vavlukis M, Georgieviska-Ismail LJ, Bosevski M, Borozanov V. Predictors of in-hospital morbidity and mortality in patients with coronary artery disease treated with coronary artery bypass surgery. Prilozi. 2006; 27 (2): 87-113.

5. Lawrence DR, Valencia O, Smith EEJ, Murday A, Treasure T. Parsonnet score is a good predictor of the duration of intensive care unit stay following cardiac surgery. Heart. 2000; 83: 429-32.

6. Tuman KJ, McCarthy RJ, March RJ, Najafi H, Ivanikovich AD. Morbidity and duration of ICU stay after cardiac surgery: a model of preoperative risk assesment. Chest. 1992; 102 (1): 36-44.

7. Garner JS, Jarvis WR, Emori TG, Horan TC, Hughes JM. CDC definitions for nosocomial infections, 1988. Am J Infect Control. 1988; 16 (3): 128-40.

8. Geissler HJ, Holzl P, Marohl S, Kuhn-Réginer F, Mehlhorn U, Südkamps M, et al. Risk stratification in heart surgery: comparison of six score systems. Eur J Cardiothorac Surg. 2000; 17: 400-6.

9. Bernstein $\mathrm{AD}$, Parsonnet $\mathrm{V}$. Bedside estimation of risk as an aid for decisionmaking in cardiac surgery. Ann Thorac Surg. 2000; 69: 823-8.

\section{Potencial Conflito de Interesses}

Declaro não haver conflito de interesses pertinentes.

\section{Fontes de Financiamento}

O presente estudo não teve fontes de financiamento externas.

\section{Vinculação Acadêmica}

Este artigo é parte de tese de Doutorado de Tânia Mara Varejão Strabelli pela Faculdade de Medicina da Universidade de São Paulo.

10. Rosenfeld R, Smith JM, Woods SE, Engel AM. Predictors and outcomes of extended intensive car unit length of stay in patients undergoing coronary artery bypass graft surgery. J Card Surg. 2006; 21: 146-50.

11. Parsonnet $\mathrm{V}$, Dean D, Bernstein AD. A method of uniform stratification of risk for evaluating the results of surgery in acquired adult heart disease. Circulation. 1989: 79 (6 Pt 1): 3-12.

12. Wynne-Jones K, Jackson M, Grotte G, Bridgewater B. Limitations of the Parsonnet score in measuring risk stratification in the north west of England. The North West Regional Cardiac Syrgery Audit Steering Group. Heart. 2000; 84: 71-8.

13. Berman M, Stamler A, Sahar G, Georghiou GP, Sharoni E, Brauner R, et al. Validation of the 2000 Berstein-Parsonnet score versus EuroSCORE as a prognostic tool in cardiac surgery. Ann Thorac Surg. 2006; 81: 537-40.

14. Nashef SAM, Roques F, Michel P, Gauducheau E, Lemeshow S, Salamon R. European system for cardiac operative risk evaluation (EuroSCORE). Eur J Cardiothorac Surg. 1999; 16: 9-13.

15. Ad N, Barnett SD, Speir AM. The performance of the EuroSCORE and The Society of Thoracic Surgeons mortality risk score: the gender factor. Interact Cardiovasc Thorac Surg. 2007; 6: 192-5.

16. Sanchez Velasquez LD, Reyes Sanches ME, Carrillo Rojas JA, Rincon Salas J, Medina Villaverde JC, Venegas Alvarez D. Prediccion de mortalidad en enfermos operados de corazon: estudio comparative de cuatro modelos predictivos. Rev Asoc Mex Med Crit y Ter Int. 2003; 17 (1): 19-23.

17. Cortina Romero JM. Scores de gravedad y complejidad en cirurgia cardíaca: usos y limitaciones. Rev Esp Cardiol. 2005; 58 (5): 473-6. 